\title{
Dexmedetomidine: the answer for difficult cases?
}

Ferreira $\mathbf{S 1}$, Dias J1, Alves J1.

Anesthesiology Department of Hospital Sra da Oliveira, Guimarães, Portugal

\section{BACKGROUND}

Deep sedation or general anesthesia (GA) with benzodiazepines, barbiturates or propofol may cause respiratory depression and abolition of airway protective reflexes.

Dexmedetomidine ( $\alpha 2$ selective-agonist) - Sedative, anxiolytic and non-respiratory depressant properties

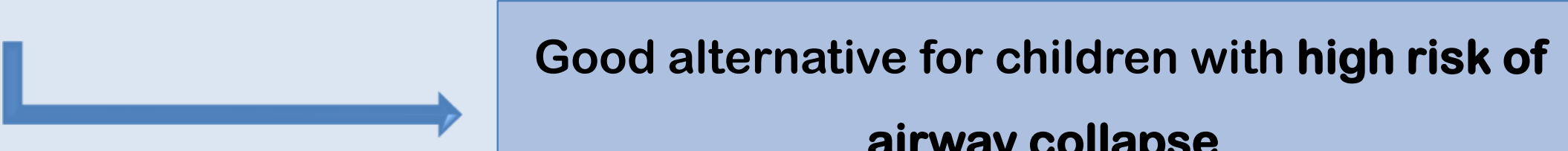

\section{airway collapse}

\section{CLINICAL CASE}

$\checkmark 18$ months male, $13 \mathrm{Kg}$, ASA 3, laryngomalacia suspected;

$\checkmark$ PROPOSED PROCEDURE: Cranioencephalic and neuroaxis MRI

$\checkmark$ History of macrocephaly, delayed psychomotor development, kyphoscoliosis and history of recurrent respiratory infections.

- Administration of midazolam bolus $0.05 \mathrm{mg} / \mathrm{kg}$ and dexmedetomidine $2 \mu / \mathrm{kg}$ in 10 minutes $\rightarrow$ score 4 of the Ramsay sedation scale was reached.

- Dexmedetomidine perfusion was started at $1 \mu / \mathrm{kg} / \mathrm{h}$.

- Extra bolus administration of midazolam $0.05 \mathrm{mg} / \mathrm{kg}$ and ketamine $1 \mathrm{mg} / \mathrm{g}$.

- Maintained spontaneous ventilation and hemodynamic stability throughout the procedure.

- Discharge to home after recovery without intercurrences.

\section{DISCUSSION}

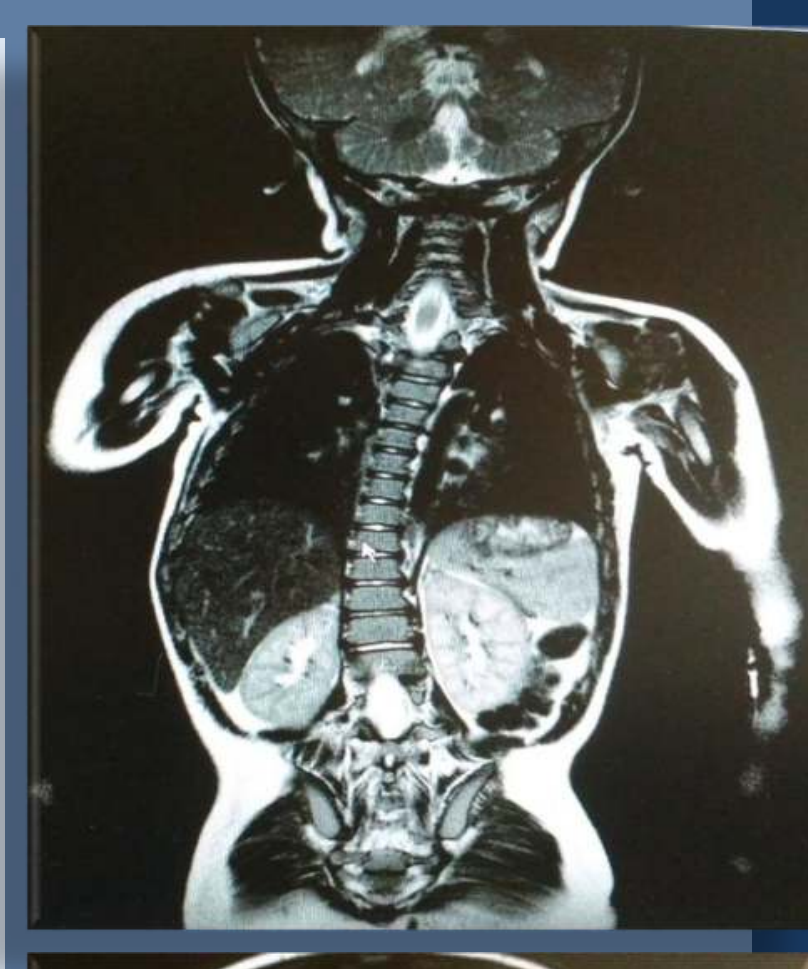

- The use of dexmedetomidine in children is not FDA approved, however its use in pediatrics has been described in the literature.

- Sedative drug that does not cause respiratory depression $\rightarrow$ attractive in children with greater risk in airway approach.

- Mason KP et al. describe an effective dexmedetomidine protocol 2-3 $\mu \mathrm{g} / \mathrm{kg}$ iv followed by na infusion up to $2 \mu \mathrm{g} / \mathrm{kg} / \mathrm{h}$ (plus adjuvant sedative drugs bolus when necessary) $\rightarrow$ bradycardia, hypotension and sinus arrhythmias were described with these doses.

- Sedation is described in combination with dexmedetomidine and ketamine with adequate levels of sedation, $\downarrow$ potential for adverse effects.

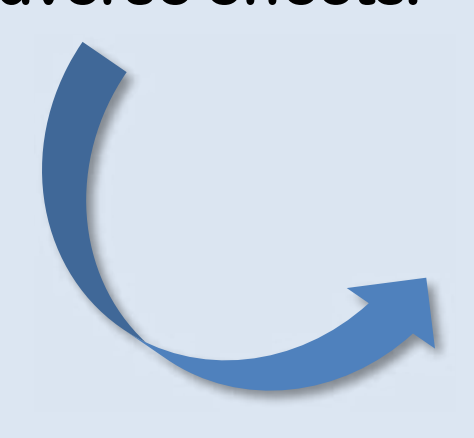

Synergistic sedative effect with opposite effects at cardiovascular level, appearing to be the key to maintaining spontaneous ventilation preserving hemodynamic stability.

\section{LEARNING POINTS}

- Sedation in remote locations is allways a challenge (specially in high risk children in airway management). 